\title{
IMPROVISIEREN ALS PERFORMATIVE MUSIKPRAXIS. ZUGÄNGE UND FORSCHUNGSPERSPEKTIVEN
}

\author{
Martin Pfleiderer
}

Seit der Jahrtausendwende zeichnet sich ein neues und wachsendes Interesse am Improvisieren ab - und das nicht nur in der Musikforschung, sondern in so unterschiedlichen Bereichen wie den Tanz- und Theaterwissenschaften, den Performance Studies, der Philosophie, Soziologie und Informatik sowie in der Politik und im Management. Indizien für dieses Interesse sind die Gründung von Zeitschriften und Forschungsnetzwerken sowie die Herausgabe von Sammelbänden zum Thema. 2004 wurde in Kanada die Online-Zeitschrift Critical Studies in Improvisation / Études critiques en improvisation gegründet. Federführend waren Theaterwissenschaftler der Universität Guelph, aus deren Kreis 2015 der Improvisation Studies Reader (Caines/Heble 2015) hervorging - ein Sammelband mit kanonischen Texten zum Improvisieren sowie Reflexionen von improvisierenden Künstlern; der thematische Schwerpunkt des Bandes liegt im Bereich der Theaterwissenschaften und Performance Studies. Im Jahre 2006 wurde in den USA die International Society for Improvised Music gegründet. 2016 erschien das zweibändige Oxford Handbook of Critical Improvisation Studies mit knapp 60 Texten aus verschiedenen Bereichen der Improvisationsforschung (Lewis/Piekut 2016a).

Improvisation wird in den Critical Improvisation Studies als grundlegendes Merkmal des menschlichen Lebens verstanden, das weit über den künstlerischen Bereich hinausweist: Improvisieren ist ein Charakteristikum fast aller menschlichen Praktiken, bei denen bestimmte körperlich verankerte Routinen immer wieder neu an die sich wandelnden situativen Gegebenheiten angepasst werden. Improvisiert wird u.a. im Gespräch und in der Alltagskommunikation, beim Kochen und bei Feiern, improvisiert wird aber auch in der Politik oder in der Unternehmungsführung. Denn das menschliche Zusammenleben, so die grundlegende Annahme, ist nicht planbar, zumindest nicht bis ins letzte Detail. Feste Pläne und unverrückbare Routinen sind in vielen Lebensbereichen auch gar nicht sinnvoll, da sie den Erfindungsreichtum und die kreativen Potentiale des menschlichen Handelns unnötig einengen. 
In der neueren Improvisationsforschung dient die musikalische Improvisation, und insbesondere die Jazzimprovisation, oftmals als ein Modellfall, an dem sich Forscher anderer Disziplinen orientieren und von dem sie allgemein etwas über den improvisatorischen Charakter sozialer und kultureller Praktiken lernen wollen. Dieses interdisziplinäre Interesse verstehe ich als Chance und zugleich als Herausforderung für die Musikforschung: Als Chance, Fragestellungen und Erkenntnisse aus dem Bereich der Musik in ein transdisziplinäres Forschungsfeld einzubringen, und als Herausforderung, theoretische und methodologische Ansätze aus anderen Wissenschaftsdisziplinen für die Musikforschung fruchtbar zu machen. Vor diesem Hintergrund möchte ich im Folgenden Einblicke in Fragestellungen, Erklärungsmodelle und Forschungsmethoden der neueren musikbezogenen Improvisationsforschung geben. Zu Beginn sollen einige zentrale Themen im Diskurs um Improvisation skizziert werden. Bisherige und mögliche zukünftige musikwissenschaftliche Zugänge zum Improvisieren werden sodann am Beispiel der Jazzimprovisation diskutiert. Dabei stellt sich auch die Frage einer übergreifenden Theorie der musikalischen Improvisation. Schließlich möchte ich einige Perspektiven einer künftigen Erforschung musikalischen Improvisierens diskutieren.

\section{Der Diskurs ums Improvisieren}

In der Musikforschung ist das Phänomen der Improvisation noch immer eine Art »kategoriale[r] Geisterfahrer « (Kaden 1993: 47): Man begegnet ihm zwar immer wieder, aber meistens steht es quer zur »eigentlichen Richtung « der Forschungsinteressen. Dessen ungeachtet gibt es inzwischen eine reichhaltige Forschungsliteratur zur musikalischen Improvisation, die von Jahr zu Jahr weiter anwächst. Vor allem Musikethnologen und Jazzforscher interessieren sich seit Jahrzehnten für Improvisationspraktiken. Historische Musikwissenschaftler beschäftigen sich mit dem Improvisieren vor allem im Kontext der historisch informierten Aufführungspraxis von Musik vor 1800 oder der aleatorischen Konzepte in der Neuen Musik. Eng damit verknüpft sind Bestrebungen, Kompetenzen der musikalischen Improvisation zu vermitteln und in die Musikerausbildung einfließen zu lassen. Definiert wird das Improvisieren dabei nahezu übereinstimmend als »spontane[s] Erfinden, Gestalten und gleichzeitige[s] Ausführen von Musik« (Ferand 1956: 5) oder als »creation of music in the course of performance (Nettl/Russell 1998) - also als ein musikalischer Schaffensprozess am Instrument während der Darbietung. Musikalische Improvisationspraktiken lassen sich demnach nicht von der performativen Si- 
tuation trennen, die u.a. durch die Wechselbeziehung zwischen Musikern untereinander und zwischen Musikern und Publikum geprägt wird. Zugleich lässt sich das Improvisieren im Sinne der neueren Praxistheorien (vgl. etwa Reckwitz 2003) durch ein spezifisches Verhältnis des Musikers zu seinem Körper und zu seinem Instrument sowie durch eine spontane Anpassung von Routinen an sich wandelnde Situationen und Rahmenbedingungen charakterisieren.

Allerdings tauchen diese Aspekte im älteren Diskurs ums Improvisieren eher am Rande auf. In der Einleitung zum Oxford Handbook of Critical Improvisation Studies nennen George Lewis und Benjamin Piekut eine Reihe von Themen und Argumentationsfiguren, die für den wissenschaftlichen Umgang mit dem Phänomen der Improvisation bislang bezeichnend waren (Lewis/ Piekut 2016b: 3-12). Ich möchte die dort genannten Themen zum Ausgangspunkt eines kurzen Überblicks über den Improvisationsdiskurs nehmen. Mein besonderes Augenmerk liegt dabei auf der musikalischen Improvisation, und ich ergänze Hinweise auf Teilaspekte und Texte, die mir in diesem Zusammenhang wichtig erscheinen, jedoch von den beiden Autoren nicht genannt werden.

Oft wird der Begriff des Improvisierens vermieden und durch andere Ausdrücke ersetzt - obwohl es de facto ums Improvisieren geht. Lewis und Piekut sprechen hier von Verschleierung (»masking «), die in weiten Bereichen der Musikethnologie anzutreffen ist, aber zum Beispiel auch in der Soziologie des Alltags. So analysiert etwa Erving Goffman ausgiebig die vielen improvisatorischen Aspekte von Alltagsinteraktionen, vermeidet dabei jedoch den Begriff des Improvisierens. Eine Ausnahme ist Pierre Bourdieu, der 1976 im Entwurf einer Theorie der Praxis den Habitus als das »durch geregelte Improvisationen dauerhaft begründete Erzeugungsprinzip« von sozialen Praktiken beschreibt (Bourdieu 1976: 170). Ein improvisatorisches Moment ist somit in den vom Habitus geprägten Praktiken von vornherein mitangelegt, was jedoch von Bourdieu nicht näher erläutert wird.

Oftmals wird Improvisation nicht nur verschleiert, sondern geleugnet oder abgewertet. Improvisieren sei nichts anderes als unbeholfenes, durch Zufälle geprägtes >Herumnudeln<, erfordere keinerlei musikalische Expertise und sei ohne künstlerischen Wert. Oder es sei eine bewusste Täuschung, da angeblich spontan improvisierende Musiker doch immer wieder nur dieselben Phrasen herunterspulen. Dass dem nicht so ist, dürfte jedem klar sein, der sich eingehender mit dem Improvisieren beschäftigt.

Weit verbreitet im Diskurs ums Improvisieren ist noch immer eine konzeptionelle Opposition zwischen Prozess und Produkt, bei der das Improvisieren dem Komponieren entgegengestellt wird. Beim Improvisieren zähle der Prozess, beim Komponieren das fertige Produkt, die Komposition. Während das 
Ziel der Komposition Perfektion sei (mit den Worten Christian Kadens: das "So-und-nicht-anders-Sein «), werde beim Improvisieren das Unvollendete, das »So-und-auch-anders « in Kauf genommen (Kaden 1993: 51f.). Der Jazzkritiker Ted Gioia spricht in diesem Zusammenhang vom Jazz als einer »imperfect art « (Gioia 1988). Oftmals wird das perfektionierte Musikwerk mit der Hochkultur in Verbindung gebracht, Improvisation und Imperfektion dagegen mit untergeordneten Kulturbereichen. In diese ästhetische Opposition zwischen angeblicher Unvollkommenheit und angeblich vollkommener Kunst werden auch die Musiker verstrickt, die Kompositionen aufführen und interpretieren. Denn auch bei der Aufführung von Kompositionen zählt ja seit dem 19. Jahrhundert zunehmend die Perfektion im Sinne der >Treue zum Notentext. Dass gerade durch die Personalunion im Erfinden und Ausführen von Musik besondere musikalische Qualitäten entstehen können, die bei einer Arbeitsteilung zwischen Komponist und Musiker nicht möglich sind (vgl. Wilson 1994: 67), wurde allerdings bislang nur selten thematisiert.

Ein weiteres Thema, das sich vor allem mit dem Improvisieren im Jazz verbindet, ist die durchaus soziopolitisch verstandene Verknüpfung von Improvisation mit Freiheit und Befreiung. Das Zusammenspiel in einer Jazzband wird als Symbol für eine ungezwungene, demokratische Kooperation unter freien Menschen verstanden, oder aber es wird auf die Verbindungen von musikalischen Improvisationspraktiken und der gesellschaftlichen Emanzipation der Afroamerikaner hingewiesen (vgl. Lewis 1996, Monson 2007).

Die Freiheit des Improvisierens hängt wiederum eng mit der Ereignishaftigkeit, Momenthaftigkeit, Emergenz und Undeterminiertheit der Musik und mit der Spontaneität und Kreativität der Musiker zusammen. Die Rede ist von einer Feier des Moments (>celebration of the moment<), oder kurz: von der >Andersartigkeit< des Improvisierens - im Gegensatz zur Wiederholung des Bekannten und Geplanten. Undeterminiertheit wird dabei oftmals auf das Fehlen einer schriftlichen Spielvorlage bezogen, aber auch auf den Verzicht eines vorab fixierten Planes oder Spielentwurfs. Spontaneität meint ein ungeplantes Handeln, das in erster Linie aus einem inneren, momentanen Impuls oder aus der Inspiration der Situation heraus erfolgt.

Allerdings weiß jeder Musiker, dass das Improvisieren nicht so voraussetzungslos ist, wie es die Topoi von der Freiheit und Ereignishaftigkeit vielleicht suggerieren. Improvisieren ist eine Expertise, die gelernt sein will. Sie beruht auf einer umfassenden Wissensbasis, die sich die Musiker in jahrelangen Prozessen des Lernens, Übens und Spielens aneignen. Dabei lassen sich drei Aspekte unterschieden (vgl. hierzu Nettl 1974): Grundlegend ist zunächst das Aneignen oder > Erspielen< eines Vokabulars an Bausteinen, Mustern oder For- 
meln durch den Musiker. Manche dieser Bausteine fließen unverändert in bestimmte Improvisationen ein, in der Regel werden sie jedoch im Spielprozess verändert, mit anderen Mustern kombiniert und so der jeweiligen Spielsituation angepasst. Diese situativen Veränderungen und Transformationen folgen bestimmten Strategien oder Gestaltungsprinzipien, also habituellen oder gezielt und bewusst eingesetzten Techniken wie dem Paraphrasieren, Ornamentieren oder Variieren. Hinzu kommt in vielen Genres die Orientierung an bestimmten musikalischen Modellen. Diese sorgen vor allem für eine zeitliche, z.T. aber auch harmonische oder melodische Strukturierung der Musik. Bestimmte Formmodelle bilden eine Art Rahmen oder Skelett für die Improvisationen, ebenso Melodievorlagen, Rhythmen (etwa Patternmodelle und Grooves) oder, wie im Jazz, die zyklisch wiederholten Harmoniefolgen eines Jazzstandards. Diese Modelle dienen insbesondere beim Zusammenspiel mehrerer Musiker als Referenzrahmen für die musikalische Gestaltung. Solche regelmäßig wiederkehrenden Aspekte des Improvisierens - Bausteine, Gestaltungsweisen und Modellbezüge - sind der wissenschaftlichen Rekonstruktion wie auch der musikpraktischen Lehre weit eher zugänglich als das Einmalige, Spontane, Ereignishafte und Emergente des Improvisierens. Sie stehen daher im Zentrum vieler analytischen Studien zur musikalischen Improvisation ebenso wie von Improvisationsanleitungen und Improvisationsschulen. Ich möchte dies am Beispiel der Jazzforschung konkretisieren.

\section{Improvisieren als Gegenstand der Jazzforschung}

Hintergrund und Zielrichtung der analytisch ausgerichteten Jazzforschung war zunächst die Würdigung der künstlerischen Qualitäten des Jazz und seine angestrebte Aufwertung als künstlerisch wertvolle Musikform. Seit den 1950er Jahren gibt es zahlreiche analytische Studien, die einzelne Jazzimprovisationen würdigen - angefangen von Gunther Schullers (1958) Analyse des Solos von Sonny Rollins in »Blue Seven« (1956) bis zu der jüngsten Buchreihe Oxford Studies in Recorded Jazz, in der einflussreiche Jazzaufnahmen analytisch beschrieben werden (Harker 2011, Waters 2011, Tackley 2012, Elsdon 2013, Solis 2014, Cooke 2017).

Allerdings wurde wiederholt auf den Umstand hingewiesen, dass bestimmte Improvisationsaufnahmen nur Indikatoren, bestenfalls prototypische Beispiele für genrespezifische oder individuelle Improvisationsstile und -praktiken sind. Nicht die einzelnen Improvisationen bzw. deren Aufnahmen, sondern übergreifende Gestaltungsprinzipien eines Musikers oder einer Stilrichtung rücken daher ins Zentrum der Improvisationsforschung. Gefragt wird 
nach den Gemeinsamkeiten in der melodischen, harmonischen, rhythmischen und klanglichen Gestaltung, nach den Bausteinen und Gestaltungsprinzipien, die für die Improvisationen eines Stilbereichs oder eines Musikers typisch sind. In einer historischen Perspektive ergänzen diese zeitlichen, regionalen oder individuellen Improvisationsstile einander zu einer Stilgeschichte der Jazzimprovisation. Dabei werden verschiedene musikanalytische Fragestellungen verfolgt und erhalten, je nach Gegenstand, unterschiedliches Gewicht. Oft liegt ein Schwerpunkt in der harmonischen Analyse, also der Frage, wie bestimmte Tonhöhen auf die Harmonik der Stückvorlage bezogen sind. Hierzu haben sich inzwischen sehr differenzierte jazztheoretische Ansätze entwickelt, die wiederum die Jazzpädagogik stark dominieren. Dagegen werden Fragen der rhythmischen und klanglichen Gestaltung eher am Rande thematisiert; womöglich ist das Methodenarsenal für diese Fragen noch nicht differenziert genug. Immer wieder geht es um die melodischen Gestaltungsweisen, manchmal auch um das konkret verwendete Repertoire an Pattern. Im Zuge der analytischen Studien von Schuller (1958) und Barry Kernfeld (1983) hat sich eine analytische Unterscheidung zwischen drei Improvisationsstrategien durchgesetzt: dem Paraphrasieren einer Melodievorlage, dem Zusammenfügen und Variieren bestimmter Bausteine oder Patterns (»formulaic improvisation «) sowie schließlich einer Improvisationsweise, bei der motivische Bezüge im Zentrum stehen.

»[P]araphrase involves the strict, bar-by-bar embellishment of pre-existing material in such a way that it remains recognizable; formulaic and motivic improvisation create new ideas. Paraphrase and motivic improvisation involve the constant development respectively of a specific theme and fragment, which give a piece a particular identity; formulaic improvisation proceeds by means of the ingenious weaving together of fragments from a general repertory that is common to many diverse pieces« (Kernfeld 2002: 320).

Bei einem stilanalytisch orientierten Analyseansatz ist es natürlich sinnvoll, auf größere Korpora von Improvisationen zurückzugreifen, im Idealfall auf alle zur Verfügung stehenden Aufnahmen. Ein beeindruckendes Beispiel hierfür ist die detaillierte Studie des Patternvokabulars von Charlie Parker, die Thomas Owens bereits 1974 veröffentlicht hat (Owens 1974). Allerdings wird der Korpus an Improvisationsaufnahmen, die der Analysierende kennt und gehört hat, in der Regel nicht explizit ausgewertet, sondern fließt als implizite Hörerfahrung in dessen Vorwissen ein. Im Zentrum der Analyse stehen stattdessen einzelne, als repräsentativ oder prototypisch empfundene Improvisationen, durch deren exemplarische Analyse die typischen Gestaltungskriterien eines Musikers verdeutlich werden (Jost 1975: 15f.). 
Lange Zeit hat sich die Jazzforschung auf die Untersuchung von begleiteten Improvisationen einzelner Musiker, sog. Jazzsoli, konzentriert. Erst in jüngerer Zeit werden Aspekten der Interaktion innerhalb eines Ensembles vermehrt Aufmerksamkeit geschenkt, also nicht nur die Improvisationsstimme des Solisten, sondern darüber hinaus deren Bezüge zum Spiel der Rhythmusgruppe analysiert. Voraussetzung hierfür sind umfangreiche Partiturtranskriptionen, wie sie in den Studien von Paul Berliner (1994), Ingrid Monson (1996), Richard Hodson (2007) oder Keith Waters (2011) zu finden sind. Benjamin Givan (2016) hat allerdings kritisiert, dass der Begriff der >musikalischen Interaktion< oftmals eher unpräzise verwendet wird. Er plädiert dafür, zwischen drei verschiedenen Typen der Interaktion zu unterschieden: »Microinteraction « meint demnach eine Feinkoordination hinsichtlich des Tempos und des Timings, der Dynamik, der Intonation und Artikulation im Ensemble; solche Interaktionsprozesse gibt es in allen Musikgenres, in der >Klassik ebenso wie im Rock, sie sind somit nicht an das Konzept des Improvisierens gebunden. »Macrointeraction« bezieht sich dagegen auf übergeordnete Textur- und Strukturwechsel sowie koordinierte dramaturgische Entwicklungen: Die ganze Band spielt beispielsweise sukzessive lauter oder dichter oder wechselt ihre Begleitpatterns - was von den Musikern in vielen Musikgenres, z.B. im Blues oder Rock, auch ohne ausgefeilte improvisatorische Expertise praktiziert werden kann. Dagegen konzentrieren sich viele Studien zur Interaktion im Jazz auf die verschiedenen Formen der »motivic interaction«. Darunter versteht Givan das Aufgreifen, Weiterspinnen und Verändern von melodischen, rhythmischen oder harmonischen Zellen. Er erläutert anhand zahlreicher Jazzaufnahmen verschiedene Möglichkeiten der motivischen Interaktion, weist allerdings auch darauf hin, dass nicht alle Jazzmusiker diese Interaktionsform favorisieren. Viele Jazzmusiker sprechen sich sogar ausdrücklich gegen eine motivische Interaktion zwischen den Musikern aus: Der improvisierende Solist soll nicht durch unerwünschte Einwürfe und Interaktionsangebote der Begleitmusiker von seinem Solo abgelenkt werden. Vielmehr bevorzugen manche Jazzgruppen inszenierte Performances, bei denen, ähnlich wie in nichtimprovisatorischen Genres, Abläufe zuvor geprobt und einstudiert werden. Spontane motivische Interaktionen fehlen hier völlig. Givan vermutet, dass im Hintergrund der Konjunktur der Interaktionsanalyse in der Jazzforschung auch die Verknüpfung von Jazz mit einer gerade im Jazzjournalismus verbreiteten »collectivist popular ideology« des kollaborativen, gleichberechtigten, quasi demokratischen Handelns stehen könnte.

Ein Sonderfall ist die Interaktion zwischen Musikern und Publikum. Hierzu gibt es für den Bereich des Jazz bislang nur wenige empirische Studien. Eine Befragung der Londoner Jazzposaunistin und Musikwissenschaftlerin Gail 
Brand kommt zu dem Ergebnis, dass ein konzentriert zuhörendes Publikum die Inspiration der Improvisatoren beflügelt und sie in ihrem Einfallsreichtum anspornt (Brand et al. 2012). ${ }^{1}$

In der Jazzforschung wurde erst relativ spät damit begonnen, die Musiker zu ihren Improvisationspraktiken zu befragen und diese Interviews systematisch auszuwerten. Wegweisend war die Studie des US-amerikanischen Musikethnologen Paul Berliner (1994), der in den 1980er Jahren 52 Musiker der New Yorker Jazzszene zu ihren Lern- und Improvisationsstrategien befragte. Berliner beabsichtigte mit seiner Studie, »to present artists in the light by which their own community has always appreciated them - as knowledgeable, articulate, exacting practioners of a highly valued art form « (Berliner 1994: 9) und »to increase the abilities of readers to comprehend jazz in much the same terms as do its improvisers « (ebd.: 15). Es folgten weitere Studien, u.a. von Ingrid Monson (1996). Jüngst wurde in zwei deutschsprachigen Interviewstudien (Figueroa-Dreher 2016, Müller 2017) auf Methoden der interpretativen Sozialforschung zurückgegriffen. Als besonders ertragreich haben sich in diesen beiden Studien eine Kombination von Einzelinterviews mit Musikern und Gruppendiskussionen mit ganzen Bands erwiesen. Die Gruppendiskussionen wurden direkt im Anschluss an ein Konzert (bei Müller) bzw. an Studioaufnahmen (bei Figueroa-Dreher) durchgeführt, die Tondokumente wurden als zusätzlicher Stimulus für die Befragungen verwendet. Die Leitfrage bei Christian Müller ist das Erleben des Improvisierens durch den Musiker, wobei zwischen den drei Dimensionen Musiker und Instrument, dem Zusammenspiel als Band und der Konzertsituation unterschieden wird. Silvana FigueroaDrehers Ziel ist es, eine soziologische Handlungstheorie zu formulieren, bei der von einer Gleichzeitigkeit von Entwerfen und Handeln, also einem »entwerfenden Handeln« oder »handelnden Entwerfen« (Figueroa-Dreher 2016: 72) ausgegangen wird, und diese am Beispiel des musikalischen Improvisierens empirisch zu begründen. Bemerkenswert an beiden Studien ist der hohe Reflexionsgrad und die große Differenziertheit der Aussagen der befragten Musiker.

1 Der libanesische Musikethnologe und Buzuki-Spieler Ali Jihad Racy hat nachdrücklich darauf hingewiesen, dass für das Gelingen von Improvisation in arabischer Musik (im solistischen Taqsim) die Aufmerksamkeit und Kennerschaft des Publikums ganz zentral sind. Dies kann so weit gehen, dass Musiker ausdrücklich mit erhelltem Zuschauerraum auftreten, um die Reaktionen des Publikums sehen und darauf reagieren zu können (Racy 1998: 96). 


\section{Zu einer Theorie der musikalischen Improvisation}

Bislang haben vor allem Musikpsychologen versucht, den Prozess des Improvisierens auf eine allgemeingültige Weise zu beschreiben. Von besonderem Interesse für die Psychologen ist dabei die tendenzielle kognitive Überforderung des improvisierenden Musikers, die aus dem Entscheidungsdruck in der Spielsituation entsteht. Wie gelingt es dem Musiker, die äußerst komplexe Aufgabe des Improvisierens so zu handhaben, dass kreatives Handeln möglich wird? Eine psychologische Antwort hierauf ist die Annahme einer Automatisierung bestimmter Spielabläufe, etwa durch die Wiederholung von Patterns. Ein weiterer Erklärungsansatz betont die kognitiven Verknüpfungen verschiedener Aspekte des Improvisierens, die sich mit zunehmender Spielerfahrung und Übung immer weiter differenzieren. Beide Strategien entlasten das kognitive System und ermöglichen es, dass die Aufmerksamkeit flexibel zwischen verschiedenen Aspekten des Spielprozesses - dem eigenen Spiel und dem Spiel der Mitmusiker, der Auftrittssituation, übergreifenden Stimmungen und Intentionen u.a. - hin und her wandern kann. Es ist hier nicht der Rahmen, die Vorzüge und Nachteile der verschiedenen psychologischen Improvisationsmodelle von Jeff Pressing (1988), Klaus-Ernst Behne (1992), Andreas Reinhard (1996) oder Philip Johnson-Laird (2002) en détail zu diskutieren (vgl. hierzu Figueroa-Dreher 2016: 46-57). Allerdings wird nicht ganz klar, auf welcher Erfahrungsgrundlage die Autoren ihre Theoriemodelle eigentlich formulieren. Zumindest liegen ihnen keine systematischen Datenerhebungen, sondern eher verstreute Beobachtungen zugrunde. Viele der in den Modellen gemachten Hypothesen lassen sich zudem kaum oder nur sehr schwer empirisch überprüfen.

Figueroa-Dreher hat auf zwei weitere Schwachstellen der psychologischen Improvisationstheorien hingewiesen. Zum einen konzentrieren sie sich auf den einzelnen Musiker und verweisen interaktive Aspekte des Improvisierens in den Bereich von > Randbedingungen . Außerdem tendieren die Modelle zu einem zeitlichen Auseinanderziehen von mental imaginierten Handlungsentwürfen und deren Umsetzung am Instrument. Besonders deutlich wird dies etwa bei Klaus-Ernst Behne (1992), der das Improvisieren als eine Kette von Entwerfen, Entscheiden, Handeln und Evaluieren beschreibt. Laut der gängigen Definitionen liegt der Kern des Improvisationsphänomens jedoch gerade in der Gleichzeitigkeit von Erfinden und Ausführen, von >creation< und >performance<, von Handlungsentwurf und Handlungsausführung. Diesen Aspekt 
rückt Figueroa-Dreher ins Zentrum des von ihr vorgeschlagenen Improvisationsmodells. Sie hat in ihrer Studie die Musiker zweier Flamenco-Ensembles und dreier Free-Jazz-Trios (u.a. des renommierten Schlippenbach-Trios und eines Trios um den Berliner Schlagzeuger Michael Griener) ausführlich befragt und sodann mit qualitativen Methoden der sog. datengeleiteten Theoriebildung (Grounded Theory) eine in diesen Musikeraussagen fundierte Theorie des Improvisierens formuliert.

Figueroa-Drehers Modell umfasst vier Dimensionen, die zueinander in enger Wechselbeziehung stehen: die Dimensionen des musikalischen Materials, der Interaktion, der Haltung des Musikers sowie der emergenten Musik. Gemeinsam bedingen diese Dimensionen das Ausmaß von »Improvisation« als »creativity in performance «, also den Grad der Neuheit einer Darbietung und der damit zusammenhängenden Kreativität der Musiker (s. Abb. 1).

\section{Improvisationsmodell Figueroa-Dreher}

1. Musikalisches Material

2. Interaktion

3. Haltung des Handelnden

4. Musik
(†) Formbarkeit/

- Vielfältigkeit

( Intensität

(

(+)

- „Offenheit"

(t)

$\Theta$ Emergenzgrad

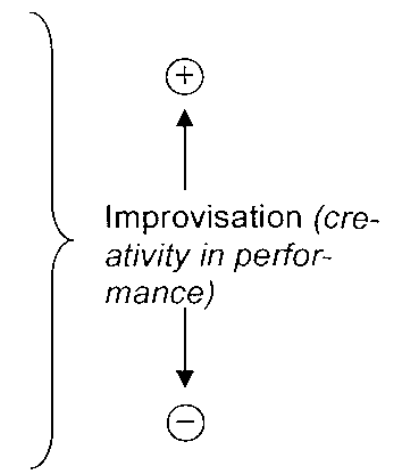

Abb. 1: Improvisationsmodell nach Figueroa-Dreher (2016: 168).

Allgemein versteht man unter >Material< Stoffe, die Gegenstand der Weiterverarbeitung und Gestaltung sind. Im Falle von Musik, so Figueroa-Dreher, wird das Material (Klänge, Geräusche, Töne, Tonfolgen, Rhythmen usw.) erst im Prozess des künstlerischen Handelns erzeugt. Musiker verfügen über Material, insofern sie über ein Wissen und Können der Materialerzeugung und Materialgestaltung am Instrument verfügen. Dabei kann Wissen als »subjektiv sedimentierte Erfahrung (Figueroa-Dreher 2016: 171), also als Ergebnis vorherigen Handelns, aber auch des Imaginierens und Fantasierens verstanden werden. Je nach Genre, Spielerfahrung, Wissensbreite und Expertise verfügen Musiker über unterschiedliches und unterschiedlich viel Material. Ein Großteil des Materials wird allerdings von den Musikern eines Genres kollektiv geteilt; sie schöpfen aus dem gemeinsamen Materialschatz von bestimmten Musiktraditionen. Material wird zumeist mit Modifikationen aktualisiert, also 
während des Spielens der jeweiligen Spielsituation angepasst. Da diese spontane Formung und Gestaltung des Materials das zentrale Merkmal des Improvisationsprozesses darstellt, ist die Formbarkeit des Materials eine wichtige Voraussetzung für das Improvisieren. Verschiedenartiges musikalisches Material besitzt unterschiedliche Grade der Formbarkeit sowie abhängig vom Musikgenre eine unterschiedlich große Vielfalt und Differenziertheit. Bisweilen wird auch während des Improvisationsprozesses neues Material sentdeckt und dann beim Üben, aber auch in der Darbietungssituation erkundet und erarbeitet. Wichtig hierbei ist das Hören - einerseits das Zuhören und das Wiedererkennen von Material im eigenen Spiel und im Spiel der anderen Musiker, andererseits das Vorweghören, die auditive Imagination.

Die Interaktion zwischen den Musikern zeichnet sich durch unterschiedliche Grade der Enge oder Intensität aus. Wenn in einem Genre, wie etwa im Flamenco, eine klare Rollenverteilung zwischen improvisierenden Solisten und den begleitenden Musikern vorgesehen ist, so ist die Intensität der Interaktion eine andere als z.B. in der freien, idiomatisch nicht gebundenen Gruppenimprovisation. Dabei umfasst die Dimension der Interaktion sowohl die Feinjustierung im Sinne von Givans »microinteraction«, die Koordination gröBerer Entwicklungen (»macrointeraction«) als auch motivische Interaktionen.

Mit der Dimension der Haltung meint Figueroa-Dreher den »Bezug der Handelnden zur Kontingenz in der Spielsituation, die Bereitschaft, Unvorhergesehenes, Ungeplantes und Zufälliges in ihr Handeln aufzunehmen und in die Musik, die sie spielen, zu integrieren. Diese Haltung kann offener (höhere Bereitschaft) oder geschlossener (niedrigere Bereitschaft) sein« (ebd.: 268). Die Offenheit zeigt sich in der Abwesenheit von Plänen und in einer Bereitschaft, spontan auf das Spiel der anderen Musiker, aber auch auf das eigene Spiel Bezug zu nehmen. Weitere Aspekte dieser Offenheit sind eine nichtreflexive Einstellung, ein Aufgeben der Situations-, Impuls- und Handlungskontrolle sowie selbstzensierender Instanzen, was auch das Zulassen automatisierter motorischer Abläufe miteinschließt. Dies geht einher mit dem Erleben eines entspannten Zustandes und einer spezifischen Konzentration und Wachheit im Hier und Jetzt.

Überraschend bei Figueroa-Drehers Modellentwurf ist vielleicht, dass die Musik selbst als weitere Dimension auftaucht. Der Grad der Emergenz bezieht sich auf die Neuheit und Lebendigkeit der Musik, die sich über bereits Bekanntes hinwegsetzt. Der Emergenzgrad ist abhängig von dem genrespezifischen Grad der Formbarkeit des musikalischen Materials und der Offenheit der Spielmodelle, vom Interaktionsgrad und von den Haltungen der Musiker. 
Die kollektiv gespielte Musik ist dabei immer mehr als die Summe der Spielstränge der einzelnen Musiker. Und sie wirkt in unterschiedlichem Grade auf den Spielprozess zurück.

Das von Figueroa-Dreher anhand ihrer Musikerbefragungen entwickelte Improvisationsmodell verlangt geradezu nach einer empirischen Überprüfung und Verfeinerung anhand vergleichender Studien zwischen unterschiedlichen Improvisationsgenres. Obwohl sich Figueroa-Dreher auch auf Aussagen der Flamenco-Musiker stützt, hat ihr Modell womöglich durch die Dominanz der Free-Jazz-Musiker eine gewisse Schlagseite in Richtung freier, kollektiver Gruppenimprovisation. Wie verhält es sich mit den vier Dimensionen ihres Improvisationsmodells in anderen Musikgenres? Und welche Aspekte lassen sich auf nicht-musikalische Improvisationspraktiken übertragen? Bedenkenswert ist zudem Figueroa-Drehers (ebd.: 315) Vorschlag, in der Improvisationsforschung verschiedene Ansätze und Methoden interdisziplinär zu kombinieren. Während die Untersuchung des musikalischen Materials und des emergenten Charakters der Musik eine musikanalytische Herangehensweise erfordere, lassen sich Phänomene der Interaktion und der Improvisationshaltung eher mit den empirischen Methoden der Sozial- und Verhaltenswissenschaften untersuchen.

\section{Methodologische Perspektiven der Improvisationsforschung}

Grob gesehen lassen sich in der Forschung zur musikalischen Improvisation zwei empirische Herangehensweisen unterscheiden: Die Analyse von dokumentierten Improvisationen einerseits, die im weitesten Sinne ethnographische, qualitative Erforschung von Improvisationspraktiken andererseits. Innerhalb der Musikanalyse gibt es wiederum zwei Fragerichtungen: erstens die Frage nach den Besonderheiten einzelner Improvisationen, also nach den emergenten Charakteristika der Musik, und zweitens die Materialanalyse, also die Beschreibung und Analyse von klanglichen Mustern und Bausteinen sowie, abhängig von genretypischen Modellen und Konventionen, den Strategien der Formung und Gestaltung dieses Materials. Beide Fragerichtungen sind aufeinander bezogen: Einerseits lässt sich die Neuheit und Emergenz konkreter Improvisationen nur mit der Kenntnis der in einem Genre üblichen Materialbasis und Gestaltungskonventionen sinnvoll beurteilen. Andererseits muss die Untersuchung des Materials und der Gestaltungsstrategien ihren Ausgangspunkt in der Analyse konkreter Improvisationen nehmen. Je größer der Fundus an Improvisationen, umso eindeutiger lassen sich Rückschlüsse auf das 
musikalische Material und die Gestaltungsprinzipien ziehen, das bzw. die typisch für einen Musiker, ein Ensemble oder ein Improvisationsgenre sind.

Grundlage des musikanalytischen Ansatzes sind Höranalysen sowie Transkriptionen von Tonaufnahmen. Die Problematik von Transkriptionen ist in der Musikethnologie (und Popularmusikforschung) bereits ausführlich thematisiert worden. Im Gegensatz zu präskriptiven, also zum Nachspielen gedachten Transkriptionen, sind analytische oder deskriptive Transkriptionen immer an konkreten analytischen Fragestellungen orientiert. Sie erheben keinen Anspruch, objektive Repräsentationen der Musik zu sein, sondern sind immer an den Höreindruck des Analysierenden sowie an dessen Erkenntnisinteressen und Hörerfahrungen geknüpft. Zugleich sind Transkriptionen vielfach Gratwanderungen zwischen den Kriterien der leichten Lesbarkeit und der sachlichen Angemessenheit.

Der immense Aufwand von vergleichenden Korpusstudien, also der Untersuchung von möglichst vielen Aufnahmen bzw. deren Transkriptionen, kann womöglich durch neue, computergestützte Methoden bewältigt werden. Besonders vielversprechend erscheint mir die Kombination einer herkömmlichen Analyse einzelner exemplarischer Improvisationen mit der statistischen Auswertung solcher Korpora, also mit einem > distant listening < im Sinne des Konzepts des »distant reading « des Literaturwissenschaftlers Franco Moretti (2013). Hierzu gibt es erste Ansätze, so das Jazzomat Research Project mit der Weimar Jazz Database, einer computerlesbaren Datenbank von 456 Jazzsoli (http: / /jazzomat.hfm-weimar.de/, Pfleiderer et al. 2017) oder einer Datenbank von Flamencoaufnahmen (http://www.cofla-project.com/, Kroher et al. 2016). Ob Improvisationsaufnahmen in absehbarer Zeit verlässlich automatisch transkribiert bzw. direkt als Audio-Daten statistisch ausgewertet werden können, ist angesichts der zahlreichen technischen Probleme eher unwahrscheinlich, angesichts der rasanten Entwicklungen im sogenannten Music Information Retrieval jedoch nicht ausgeschlossen. ${ }^{2}$

Was bei einer Musikdarbietung improvisiert, was geplant und reproduziert ist, lässt sich allerdings aufgrund des bloßen Hörens nie mit Sicherheit entscheiden - das wissen nur die Musiker selbst. Daher sind Befragungen von Musikern und die gewissenhafte inhaltsanalytische Interpretation dieser Interviews und Gruppendiskussionen wichtig, ja unausweichlich. Nur auf diese Weise ist etwas über die interaktive Dimension, die Spielhaltung, die Motivation und das Erleben von Improvisationspraktiken herauszufinden.

2 In diese Richtung zielt das transatlantische Forschungsprojekt Dig That Lick. Analysing large-scale data for melodic patterns in jazz performances (http: / / digthat-lick.eecs.qmul.ac.uk/). 
Zwar finden sich in den Interviewstudien von Berliner (1994) und Monson (1996) auch viele Transkriptionen und analytische Abschnitte, durch die die Musikeraussagen veranschaulicht und erläutert werden. Allerdings beziehen sich die befragten Musiker nicht explizit auf eigene, konkrete Improvisationen. Figueroa-Dreher (2016) hat zwar Musikaufnahmen gemacht, die sie ihren Interviews mit den Musikern als Stimulus zugrunde legte. Konkrete musikalische Sachverhalte erwähnt sie jedoch in ihrer Arbeit nur am Rande, Müller (2017) blendet sie völlig aus. Für Musikforscher stellt sich natürlich die Frage: Wie lassen sich Musikerbefragungen und Musikanalyse sinnvoll kombinieren? Der englische Musikpsychologe John Sloboda machte hierzu bereits 1985 den folgenden Vorschlag:

»By recording an improvisation and then playing it back to the performer, with as many pauses and backtracks as required, we could hope to obtain a detailed record of the conscious decisions involved in constructing the improvisation « (Sloboda 1985: 148).

Von einer solchen Befragung entlang der konkret gespielten und durch Aufnahmen und Transkriptionen dokumentierten Musik sind nicht nur anschauliche Einsichten in die genrespezifischen oder individuellen Umgangsweisen mit dem musikalischen Material und in improvisatorische Schaffensprozesse einschließlich ihrer interaktiven Momente zu erwarten, sondern auch eine Konkretisierung der oft eher allgemein gehaltenen persönlichen Erfahrungen und Reflexionen der Musiker. Diese Idee einer sstudy of in vivo improvisation wurde jedoch bislang nur selten umgesetzt (vgl. dazu aber den Beitrag von Back und Klose in diesem Band). Martin Norgaard folgte in seiner Dissertation dem Vorschlag von Sloboda und bat sieben Jazzmusiker, begleitet von einem Drum-Loop über einen Blues in $\mathrm{F}$ zu improvisieren und direkt anschließend eine automatisierte Transkription des gerade Gespielten zu kommentieren (Norgaard 2008). Dabei wurde deutlich, auf welche musikalischen Aspekte die einzelnen Musiker während des Improvisierens jeweils ihre Aufmerksamkeit richteten. Ungeachtet unterschiedlicher individueller Gewichtungen berichteten alle Musiker sowohl von einer Bewertung und Kontrolle des gerade Gespielten als auch von dem mehr oder weniger konkreten Planen des kommenden Spiels. Generell kommen vier Strategien zur Geltung: die Verwendung gelernten musikalischen Materials (»idea bank«), die Tonauswahl unter primär harmonischen Vorzeichen (»harmonic priority«), die Tonauswahl mit Blick auf die Gestaltung der melodischen Linie (»melodic priority«) sowie schließlich der Bezug auf das Zuvor-Gespielte (»recall«). Allerdings entsprach die Spielsituation der Musiker nur bedingt der normalen Spielsituation eines Jazzmusikers, denn es fehlten die interaktiven und performativen Aspekte. 
Zudem ist fraglich, ob die von Sloboda und Norgaard favorisierte Fokussierung auf bewusste Spielentscheidungen zielführend ist - vielleicht sind ja eher körper- und instrumentengebundene Umgangsweisen mit dem erspielten musikalischen Material relevant für das Improvisieren der Musiker. So beschreiben etwa die von Müller und Figueroa-Dreher interviewten Musiker das Improvisieren als Sich-Zurücknehmen und Raumgeben, als einen fast schlafwandlerischen Zustand, in dem weder entschieden noch überhaupt nachgedacht wird, sondern in dem >die Musik < das Regime übernimmt (vgl. FigueroaDreher 2016: 252; Müller 2017: 83).

Meines Erachtens ist das Potential eines weiteren Ansatzes noch nicht voll ausgeschöpft: dem der introspektiven Selbstbeobachtung, die etwa der Studie des Soziologen David Sudnow aus den 1970er Jahren zugrunde liegt und bei der sowohl musikalische Details im Sinne der von Norgaard ermittelten Spielstrategien als auch Hinweise auf besondere körperliche und mentale Befindlichkeiten ihren Platz haben (Sudnow 1978). Neuerdings hat der Soziologe und Hobby-Jazzsaxophonist Peter Stegmaier (2012) diesen introspektiven Ansatz weiterverfolgt. Ein Problem dieses Ansatzes liegt allerdings darin, dass eine systematisch geschulte Beobachtungsgabe und ein hoher Grad an improvisatorischer Expertise wohl eher selten in einer Person zusammenfallen.

Dass bislang kaum Videoaufnahmen von Improvisations-Performances ausgewertet wurden, liegt meines Erachtens vor allem daran, dass gegenüber der akustischen Dimension die visuelle Dimension kaum nennenswerte Erkenntniszugewinne bietet. So musste Silvana Figueroa-Dreher (2016: 248ff.) feststellen, dass in den Video-Aufnahmen der von ihr untersuchten Improvisations-Ensembles kaum Interaktionen zwischen den einzelnen Musikern zu beobachten sind. Einzige Ausnahme war die von den Musikern gestisch ausgedrückte Freude über gelungene Spielmomente. Immerhin verdeutlichen die Videoaufnahmen durchweg die Haltung des konzentrierten Zuhörens, die an Gestik und Körperhaltung der Musiker ablesbar ist.

\section{Ausblick}

Der US-amerikanische Musikethnologe und Jazzforscher Gabriel Solis hat darauf hingewiesen, dass sich Improvisationsschulen und -anweisungen bislang weitgehend darauf beschränken, musikalisches Handwerk zu vermitteln. Dagegen sei das Improvisieren selbst nicht lehrbar:

»Instructional writing has generally had less to say about the creativity involved in improvising than about techniques-how, that is, to move from knowing what is possible to developing a feel for what is right in the moment. 
This is often treated as ineffable, or just something that comes with time and practice, and that therefore is not worth addressing in any detail in instructional literature « (Solis 2009: 6).

Die Kenntnis von musikalischem Material, seiner Formbarkeit und entsprechender Gestaltungsstrategien ist ohne Frage eine Voraussetzung sowohl für das Improvisieren als auch für ein umfassendes Verständnis von Improvisationspraktiken. Eine Auseinandersetzung mit dem, was erfahrene Improvisatoren über ihre Spielpraxis, ihre Spielhaltung zu sagen haben, vermag jedoch das Verständnis von Improvisationspraktiken auf instruktive Art und Weise zu erweitern. Dies gilt nicht nur für die Musiker, sondern ebenso für die Hörer. Dass ein Verständnis von Musik und musikalischen Praktiken von hoher Relevanz für die Lebendigkeit von Hörkulturen ist, gilt natürlich für jedwede Musik. Aber ganz besonders gilt dies für jene Musikrichtungen, in denen LiveDarbietungen mit improvisatorischen Gestaltungsfreiräumen und mehr oder weniger stark ausgeprägten Rückkopplungsmöglichkeiten zwischen Musikern und Hörern wesentlich sind - und für die eine schriftliche Fixierung oder technische Aufnahme und Wiedergabe nicht konstitutiv sind.

Die derzeitige Blüte der Improvisationsforschung hängt vermutlich mit der Konjunktur der Performanceforschung und der Praxistheorie innerhalb der Kultur- bzw. Sozialwissenschaften seit den 1990er Jahren zusammen. Die Anschlussmöglichkeiten gehen dabei in beide Richtungen. Eine Berücksichtigung von Konzepten und Begriffen der soziologischen Praxistheorie und der Performanceforschung, aber auch der Handlungstheorie, wie die Studie von Figueroa-Dreher zeigt, kann anregend für die musikalische Improvisationsforschung sein. Denn Aspekte der Körperlichkeit und leiblichen Ko-Präsenz von Musikern und Zuschauern, der Rolle von Artefakten - den Musikinstrumenten - für die Improvisationspraxis und der autopoietischen Feedbackschleifen innerhalb einer Performance, zwischen den Musikern sowie zwischen Musikern und Hörern, sind konstitutiv für improvisatorische Praktiken. Angesprochen sind darüber hinaus die Zusammenhänge zwischen der Offenheit der Spielsituation, der Spontaneität und Kreativität der Musiker, dem Stellenwert von implizitem Wissen und Routinen sowie schließlich eines motivational-emotionalen Wissens: Worum geht es den Musikern - und ihren Zuhörern - eigentlich beim Improvisieren? Wie die ethnographischen Studien und die Befragungen von Musikern zeigen, vermag umgekehrt eine empirische Auseinandersetzung mit musikalischen Improvisationspraktiken durchaus die soziologische und kulturwissenschaftliche Theoriebildung zu bereichern und zu verfeinern.

Die übergreifenden Gründe für das wachsende Interesse am Phänomen des Improvisierens liegen womöglich in der Erfahrung einer von zunehmender sozialer Unübersichtlichkeit und Ungewissheit geprägten Welt. Improvisieren, 
so scheint es, ist heute nicht mehr nur eine Ausnahmestrategie für jene Situationen, in denen alle Pläne und Routinen versagen, sondern sie wird in vielen Lebensbereichen zur Regel. In immer mehr Gesellschaftsbereichen sind wir gezwungen, unabhängig von Entwürfen oder verlässlichen Gewohnheiten zu handeln und dabei schnell und flexibel auf Veränderungen zu reagieren - also zu improvisieren. Vor diesem Hintergrund stellt das Improvisieren im geschützten Bereich von Musik und Kultur vielleicht ein Laboratorium für das Erproben neuartiger sozialer und kulturelle Praktiken und Handlungsweisen dar - ein Spielraum für ein gleichermaßen enges und flexibles Zusammenwirken von Menschen (Musikern, Mitmusikern und Hörern) und Dingen (Instrumenten).

\section{Literatur}

Andreas, Reinhard (1996). »Improvisation, VII. Musikpsychologie. «In: Die Musik in Geschichte und Gegenwart, Sachteil Bd. 2. Hg. v. Ludwig Finscher. Kassel u.a.: Bärenreiter u. Metzler (2. Aufl.), Sp. 595-600.

Behne, Klaus-Ernst (1992). »Zur Psychologie der (freien) Improvisation. «In: Improvisation. 10 Beiträge. Hg. v. Walter Fähndrich. Winterthur: Amadeus, S. 42-62.

Berliner, Paul F. (1994). Thinking in Jazz. The Infinite Art of Improvisation. Chicago u. London: University of Chicago Press.

Bourdieu, Pierre (1976). Entwurf einer Theorie der Praxis auf der Grundlage der kabylischen Gesellschaft. Frankfurt/M.: Suhrkamp.

Brand, Gail / Sloboda, John / Saul, Ben / Hathaway, Martin (2012). »The Reciprocal Relationship Between Jazz Musicians and Audiences in Live Performances. A Pilot Qualitative Study. «In: Psychology of Music 40 (5), S. 634-651.

Caines, Rebecca / Heble, Ajay (Hg.) (2015). The Improvisation Studies Reader. Spontaneous Acts. London: Routledge.

Cooke, Mervyn (2017). Pat Metheny. The ECM years, 1975-1984. New York: Oxford University Press.

Elsdon, Peter (2013). Keith Jarrett's The Köln Concert. New York: Oxford University Press.

Ferand, Ernest (1956). Die Improvisation in Beispielen aus neun Jahrhunderten abendländischer Musik. Mit einer geschichtlichen Einführung. Köln: Volk.

Figueroa-Dreher, Silvana K. (2016). Improvisieren: Material, Interaktion, Haltung und Musik aus soziologischer Perspektive. Wiesbaden: Springer VS.

Gioia, Ted (1988). The Imperfect Art. Reflections on Jazz and Modern Culture. New York: Oxford University Press.

Givan, Benjamin (2016). »Rethinking Interaction in Jazz Improvisation. «In: Music Theory Online 22 (3), http:/ / mtosmt.org/issues/mto.16.22.3/mto.16.22.3.givan. html (Zugriff: 22.3.2018).

Harker, Brian (2011). Louis Armstrong's Hot Five and Hot Seven Recordings. Oxford: Oxford University Press.

Hodson, Robert (2007). Interaction, Improvisation, and Interplay in Jazz. New York: Routledge. 
Johnson-Laird, Philip N. (2002). »How Jazz Musicians Improvise. « In: Music Perception 19, S. 415-442.

Jost, Ekkehard (1975). Free Jazz. Stilkritische Untersuchungen zum Jazz der 60er Jahre. Mainz: Schott.

Kaden, Christian (1993). »Vom Geist der Improvisation. «In: Ders.: Des Lebens wilder Kreis. Musik im Zivilisationsprozess. Kassel: Bärenreiter, S. 47-63.

Kernfeld, Barry (1983). »Two Coltranes. «In: Annual Review of Jazz Studies 2, S. 766.

Kernfeld, Barry (2002). »Improvisation.« In: New Grove Dictionary of Jazz, Vol. 2. London: Macmillan ( $2^{\text {nd }}$ ed. $)$, S. 313-322.

Knauer, Wolfram (Hg.) (2004). Improvisieren (= Darmstädter Beiträge zur Jazzforschung 8). Hofheim: Wolke.

Kroher, Nadine / Díaz-Báñez, José-Miguel / Mora, Joaquin / Gómez, Emilia (2016). »Corpus COFLA: A Research Corpus for the Computational Study of Flamenco Music. «In: Journal on Computing and Cultural Heritage 9 (2), Article 10.

Lewis, George E. (1996). »Improvised Music after 1950: Afrological and Eurological Perspectives. «In: Black Music Research Journal 16 (1), S. 91-122.

Lewis, George E. / Benjamin Piekut (Hg.) (2016a). The Oxford Handbook of Critical Improvisation Studies, 2 Bde. New York: Oxford University Press.

Lewis, George E. / Benjamin Piekut (2016b). »Introduction: On Critical Improvisation Studies. «In: The Oxford Handbook of Critical Improvisation Studies, Vol. 1. Hg. v. George E. Lewis u. Benjamin Piekut. New York: Oxford University Press, S. 135.

Monson, Ingrid (1996). Saying Something. Jazz Improvisation and Interaction. Chicago: University of Chicago Press.

Monson, Ingrid (2007). Freedom Sounds: Civil Rights Call out to Jazz and Africa. Oxford: Oxford University Press.

Moretti, Franco (2013). Distant Reading. London: Verso.

Müller, Christian (2017). Doing Jazz. Zur Konstitution einer kulturellen Praxis. Weilerswist: Velbrück.

Nettl, Bruno (1974). »Thoughts on Improvisation: A Comparative Approach.«In: Musical Quaterly 60 (1), S. 1-17.

Nettl, Bruno / Russell, Melinda (Hg.) (1998). In the Course of Performance. Studies in the World of Musical Improvisation. Chicago und London: University of Chicago Press.

Norgaard, Martin (2008). Descriptions of Improvisational Thinking by Artist-level Jazz Musicians. Diss., University of Texas, Austin.

Owens, Thomas (1974). Charlie Parker. Techniques of Improvisation. Diss., University of California, Los Angeles.

Pfleiderer, Martin (2004). »Improvisieren - ästhetische Mythen und psychologische Einsichten. «In: Improvisieren. Hg. v. Wolfram Knauer (= Darmstädter Beiträge zur Jazzforschung 8). Hofheim: Wolke, S. 81-99.

Pfleiderer, Martin / Frieler, Klaus / Abeßer, Jakob / Zaddach, Wolf-Georg / Burkhart, Benjamin (Hg.) (2017). Inside the Jazzomat. New Perspectives for Jazz Research. Mainz: Schott Campus.

Pressing, Jeff (1988). »Improvisation: Method and Models. «In: Generative Processes in Music. The Psychology of Performance, Improvisation, and Composition. Hg. v. John A Sloboda. Oxford: Clarendon, S. 129-178. 
Racy, Jihad Ali (1998). »Improvisation, Ecstasy, and Performance Dynamics in Arabic Music. «In: In the Course of Performance. Studies in the World of Musical Improvisation. Hg. v. Bruno Nettl u. Melinda Russell. Chicago und London: University of Chicago Press, S. 95-112.

Reckwitz, Andreas (2003). „Grundelemente einer Theorie sozialer Praktiken. Eine sozialtheoretische Perspektive. «In: Zeitschrift für Soziologie 32 (4), S. 282-301.

Schuller, Gunther (1958). "Sonny Rollins and the Challenge of Thematic Improvisation. «In: Jazz Review (November), S. 6-11.

Sloboda, John A. (1985). The Musical Mind. The Cognitive Psychology of Music. Oxford: Clarendon.

Solis, Gabriel (2009). »Introduction.«In: Musical Improvisation. Art, Education, and Society. Urbana: University of Illinois Press, S. 1-17.

Solis, Gabriel (2014). Thelonious Monk Quartet with John Coltrane at Carnegie Hall. New York: Oxford University Press.

Stegmaier, Peter (2012). »Phänomenologische und handlungstheoretische Reflektionen zum eigenen Jazzspiel als soziale Praxis. «In: Angewandte Phänomenologie. Zum Spannungsverhältnis von Konstruktion und Konstitution. Hg. v. Jochen Dreher. Wiesbaden: Springer, S. 311-342.

Sudnow, David (1978). The Ways of the Hand. Cambridge: Harvard University Press.

Tackley, Catherine (2012). Benny Goodman's Famous 1938 Carnegie Hall Jazz Concert. New York: Oxford University Press.

Waters, Keith (2011). The Studio Recordings of the Miles Davis Quintet, 1965-68. Oxford: Oxford University Press.

Wilson, Peter Niklas (1994). »Improvisation. 16 Stichworte zu einer flüchtigen Kunst«. In: Lettre International 24, S. 67-69.

\begin{abstract}
In recent years, research on improvisation has been flourishing in various disciplines such as theatre and performance studies, sociology, computer sciences, political sciences, and philosophy. In the course of this, musical practices of improvisation, above all jazz improvisation, often serve as a model for theorizing about improvisational practices in general. In this situation, musicological research on improvisation may benefit from approaches and methodologies used by other disciplines, and at the same time can transfer highly relevant insights into improvisatory music practices to the broader field of improvisation studies. After discussing both central topics of research on improvisation and recent approaches to jazz improvisation, including interaction and interviews with improvising musicians, a model of improvisation formulated by sociologist Silvana Figueroa-Dreher is sketched. Following that model a twofold methodology of future research on musical improvisation is suggested which combines an ethnographic approach towards practices of improvisation with an analysis of musical material employed in improvisatory performances.
\end{abstract}

